\title{
Perspectives
}

\section{Vaccination and established microbiological testing is essential in managing cholera and Ebola infection} \author{
Wondimagegnehu Alemu ${ }^{4}$, Mark Salter ${ }^{5}$ \\ ${ }^{1}$ Gastrointestinal Bacteria Reference Unit, Public Health England, London, United Kingdom \\ ${ }^{2}$ Central Public Health Reference Laboratory, MoHS, Lakka, Sierra Leone \\ ${ }^{3}$ Directorate of Prevention and Control, MoHS, Freetown, Sierra Leone \\ ${ }^{4}$ World Health Organisation, Country Office, Uganda \\ ${ }^{5}$ International Office, PHE, London, United Kingdom
}

Marie Anne Chattaway ${ }^{1}$, Kathie Grant ${ }^{1}$, Steve Connell ${ }^{1}$, Abdul Kamara ${ }^{2}$, Amara Jambai ${ }^{3}$,

Key words: cholera; Ebola; Sierra Leone; testing.

J Infect Dev Ctries 2015; 9(6):682-683. doi:10.3855/jidc.7158

(Received 18 May 2015 - Accepted 17 June 2015)

Copyright $\odot 2015$ Chattaway et al. This is an open-access article distributed under the Creative Commons Attribution License, which permits unrestricted use, distribution, and reproduction in any medium, provided the original work is properly cited.

In the face of the current Ebola humanitarian crisis, it is vital that endemic disease such as cholera is not forgotten. Cholera epidemics are a regular occurrence in developing African countries and in 2012 Sierra Leone had its worst cholera epidemic in 15 years. During the 2012 outbreak a dedicated cholera task force team including multiple international partners was established by the Sierra Leone, Ministry of Health and Sanitation (MoHS). The use of oral cholera vaccine (OCV) as a preventative measure against future cholera outbreaks was discussed, although at the time a decision was made against its use. Aside from the debate on how protective the OCV is [1], the major issue was effective vaccine management. The OCV generally only gives a year's protection; and two vaccine doses are required to generate this immunity. With the lack of established healthcare systems in Sierra Leone, the difficulty in ensuring mobile populations receive a second dose and the practical problems of transporting and storing a bulky vaccine with cold chain requirements, the correct application of the vaccine to ensure herd immunity was not thought to be possible. Now that the cholera epidemic in Sierra Leone is over, there is a pressing need to restart discussions on effective vaccination programme management to understand the true efficacy of such a programme. Though vaccination is not currently available for Ebola, development of a vaccination model for cholera using recently proposed strategies [2] could be used if an Ebola vaccine were to become available.

An important part of assessing the efficacy of a cholera vaccination programme and outbreak management is microbiological testing. During the outbreak, the MoHS did undertake cholera surveillance and targeted cholera hot spots for water and sanitation interventions. However, a major challenge to the accuracy of surveillance was case number ascertainment which was based on clinical assessment of symptoms rather on microbiological testing. During the outbreak, it was recognised that microbiological confirmation was essential to understanding the true burden of disease and a twoyear project was initiated to establish a diagnostic and reference laboratory for enteric bacterial infections including Vibrio cholerae, Salmonella and Shigella [3].

When Ebola cases were first recognised in Sierra Leone in 2014, risk assessments at the Central Public Health Laboratory, Sierra Leone were updated to recommend that testing for Ebola and cholera should be carried out in conjunction since the initial differential diagnosis of cholera and Ebola cannot be separated. This would enable clinical-care providers to accurately triage and treat patients accordingly (it is possible for patients to be co-infected). At the time this was not feasible due to lack of facilities but as there are now dedicated Ebola testing camps set up across Sierra Leone that could be retained as permanent 
testing centres after the outbreak, to implement a combined testing regime. The main limitation is continued funding to set up a permanent infrastructure. Though many international experts are currently in the field, it is vital that local staff are trained to enable long term sustainability. Only with strengthening the microbiological diagnosis and active cholera and Ebola surveillance in the known endemic districts will a true burden of infection, assessment of the efficacy of vaccination programmes and early detection of outbreaks be possible allowing for an early and prompt response using the current known effective interventions.

Figure 1. Cholera detection and isolation at the Central Public Health Laboratory, Lakka, Sierra Leone at containment level 2 (Musa Abu \& Fay Rhodes, MoHS, Marie Anne Chattaway, $P H E$ ). Due to a similar differential diagnosis for cholera and Ebola infection, a triaging system of samples should be implemented to rule out Ebola across the testing camps in Sierra Leone at containment level 3 before being tested for cholera.

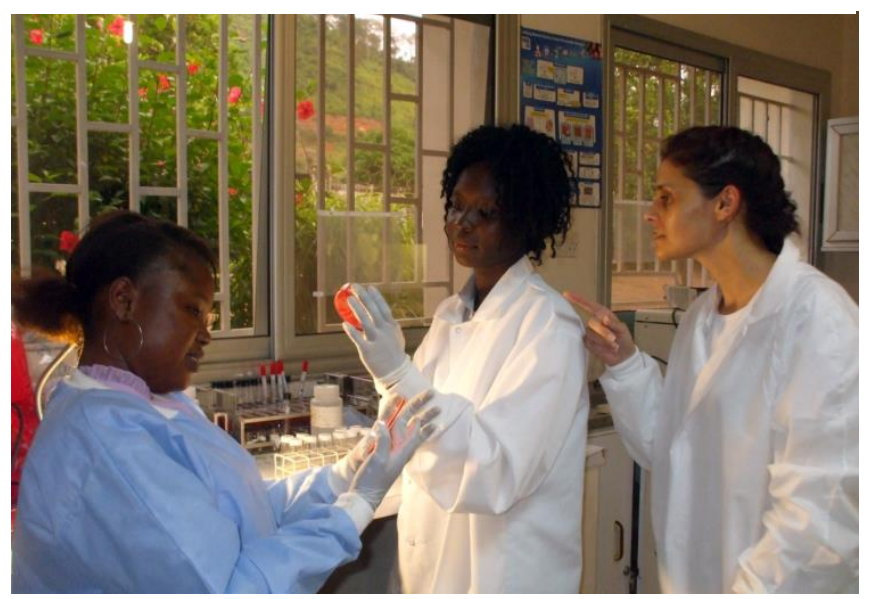

\section{References}

1. Masuet AC, Ramon Torrell JM, Zuckerman JN (2011) Review of oral cholera vaccines: efficacy in young children. Infect Drug Resist 4: 155-160.

2. Azman AS, Legros D, Lessler J, Luquero FJ, Moore SM (2015) Outbreaks of cholera in the time of Ebola: pre-emptive action needed. Lancet 385: 851-

3. Chattaway MA, Kamara A, Rhodes F, Kaffeta K, Jambai A, Alemu W, Islam MS, Freeman MM, Welfare W, Harding D, Samba AF, Abu M, Kamanda S, Grant K, Jenkins C, Nair S, Connell S, Siorvanes L, Desai S, Allen C, Frost M, Hughes D, Jeffrey Z, Gill N, Salter M (2014) Establishing an enteric bacteria reference laboratory in Sierra Leone. J Infect Dev Ctries 8: 933-941.

\section{Corresponding author}

Marie Anne Chattaway,

GBRU Clinical Scientific Lead, Deputy Head of E. coli, Shigella, Yersina and Vibrios Reference Services,

Public Health England, 61 Colindale Avenue, Colindale, London, NW9 5EQ, United Kingdom

Phone: + 02083276172

Fax:+ 02083277112

Email: marie.chattaway@phe.gov.uk

Conflict of interests: No conflict of interests is declared. 